\title{
Applying telecare in dementia: what psychiatrists need to know
}

\author{
Rajiv Siotia \& Chris Simpson
}

\begin{abstract}
Recent technological advancements have been used to help people with dementia remain independent. These are reviewed and case vignettes are used to explain their use in common scenarios. The ethical perspective and current evidence are explored in the context of telecare use.
\end{abstract}

Following diagnosis of dementia, community mental health teams aim to keep the patient in the least restrictive environment. This is often their own home. Medical interventions such as acetylcholinesterase inhibitors play a valid role in this, as do psychological interventions. However, social and environmental changes such as home care, day care and respite care can also be of enormous benefit. A number of recent advances in social care use technologies that help people with dementia remain at home for longer. This article reviews and describes these technologies, focusing on a subset of 'assistive technology' called telecare.

\section{What is 'assistive technology'?}

Assistive technology is 'any device or system that allows an individual to perform a task that they would otherwise be unable to do, or increases the ease and safety with which the task can be performed' (Royal Commission on Long Term Care, 1999). This umbrella term includes rehabilitation technologies, telecare, telehealth and electronic technology. Products range from 'low-tech' items such as walking sticks and grab rails, through mechanical and electrical equipment such as manual and powered wheelchairs, to electronic and information and communication technology (ICT) systems such as telecare. Principles of assistive technology are described in Box 1.

In dementia care, assistive technology is becoming increasingly important: for example, medicine dispensers monitor medication adherence (Case vignette 1), illuminated clocks and calendars orient patients, memory prompts and telecare enhance their independence (Box 2).

\section{Box 1 Principles of assistive technology use}

- It should be used to enable and support people with dementia to live and function more independently

- Before its use, a holistic assessment of the needs and wishes of the individual concerned should be completed

- Ethical issues should be considered in every patient. It may be particularly difficult in people with dementia; however, a detailed discussion of ethical issues should be carried out for every patient

- It should be a part of a comprehensive care package and not a replacement for human contact and care

(Integrating Community Equipment Services, 2003)

\section{Case vignette 1: a medication manager}

$\mathrm{Mr} \mathrm{A}$ is an 80-year-old man with a diagnosis of vascular dementia. He has high blood pressure and is on antihypertensive medication. He lives in sheltered housing and is independent in his activities of daily living. However, he has poor medication adherence. A dosette box was tried but it did not help.

In view of the long waiting list for home care to supervise his medication it was decided to try a medication manager. This is an electronic device similar to a dosette box, with compartments for medicines. A simple medication manager emits an audio or visual alarm at preset times to remind the patient to take a medication. More sophisticated devices can also send a message to a remote unit advising care staff of non-adherence. Mr A was given a medication manager that alerts his careworkers if he misses a dose.

Rajiv Siotia is a specialist registrar in old age psychiatry at the Friarage Hospital (Department of Mental Health, Friarage Hospital, Northallerton DL6 1JG,UK. Email: rajiv.siotia@nhs.net). Chris Simpson is a consultant old age psychiatrist in Northallerton, an associate medical director for Northallerton and Harrogate and Regional Advisor for Yorkshire. 
Box 2 Examples of assistive technology in dementia care

- Movement sensors that switch on lights

- Calendar clocks

- Memory prompts

- Medicine dispensers

- Environmental controls for remote control of appliances

- Door entry systems

- Videophones/videoconferencing

- Access to email and the internet via computer or digital interactive television

Thus, professionals can ensure that $\mathrm{Mr}$ A takes his medications with minimum restriction and intrusion into his privacy.

\section{What is telecare?}

Telecare is care provided at a distance using ICT. It has been described as the continuous, automatic and remote monitoring of real-time emergencies and lifestyle changes over time in order to manage the risks associated with independent living (Integrating Community Equipment Services, 2004).

Various terms, such 'telemedicine', 'telematics' and 'telehealth', are used interchangeably with telecare. However, the fundamental difference between telecare and telemedicine is that the former offers remote care at a person's home or place of residence, whereas the latter offers remote medical advice to the patient, wherever they may be.

If a problem arises in the patient's home, a telecare system is capable of sending an alarm to a remote centre and initiating an appropriate response. Some of the common telecare systems in use in dementia are listed in Box 3.

\section{Box 3 Examples of telecare use in dementia}

- Movement/non-movement sensors

- Falls sensors

- Flood sensors

- Fire/smoke alarm sensors

- Automatic lighting sensors

- Food/water alarm

- Window/door sensors

- Carbon monoxide sensors

- Bed/chair occupancy sensors

- Temperature range sensors

- Gas shut-off device

- Medication reminder system

\section{Box 4 Sequence of events following a fall}

1 Event: the patient falls

2 Alarm: the fall activates the sensors and an alarm is sent out

3 Call centre: receives the alarm and follows the response protocol

4 Response: this will have been agreed beforehand and might be to alert the emergency services, to telephone a carer or to call the patient to check that they are not hurt

\section{How does telecare work?}

A telecare system can be viewed as a telephone line with sensors. The type of sensor used depends on the purpose of the particular system. An event is any action that activates the sensors (Box 4). The activated sensors will generate an alarm to the call centre. The call centre acts as the hub of the telecare system with the principal roles of acknowledging an alarm, responding according to protocol and coordinating the response if needed. The response will depend on the nature of the alarm and any previously agreed actions.

Case vignette 2 illustrates the basic working of a telecare system. Although most telecare systems operate in this way, newer concepts are emerging in which, instead of a call centre, the call is sent directly to a mobile telephone or the information is logged directly onto a server which can later be viewed on a computer.

\section{Case vignette 2: fall sensors}

Mr B is an 82-year-old man with a diagnosis of dementia with Lewy bodies. He lives in his own small flat and a carer visits him daily. He is at an increased risk of falls, which is a worry for his daughter as well as the professionals involved in his care. He has had four falls in the past 6 months and on one occasion he lay on the floor for 4 hours before he was found. To allow him to continue living safely in the community, fall sensors were fitted in his home. These sensors will be activated next time Mr B has a fall and the call centre will be alerted. Once the alarm is received, the call centre will follow a pre-agreed response protocol, which might be a telephone call to check that he is fine.

\section{Generations of telecare}

As with all technology, telecare has seen rapid advances since the use of community alarms. It is now possible to group the various telecare systems into three distinct generations based on sophistication and artificial intelligence. 


\section{The first generation}

The first generation of telecare is exemplified by social alarms. These devices are installed in the home (such as pull cords or wall-mounted call units) or carried on the person. Some have minimal 'intelligence'. They must be activated by the user and they raise an alarm at a help centre or allow the individual to talk to a responder (Fisk, 2003).

It is estimated that 1.3 million people in the UK use these alarms and although they are well accepted and have been useful they have a major limitation in that the alarm needs to be initiated by the user. This may not be possible if the person has fallen or is unconscious. Alternatively, a cognitively impaired user may simply forget to initiate the call.

\section{The second generation}

Second-generation alarms are more 'proactive' and 'intelligent' as they can be activated by sensors and do not need to be physically activated by the user.

A good example is the telecare system designed to detect falls mentioned in Case vignette 2. An alternative fall detector uses one or more sensors placed on the patient's body or attached, for example, to a belt. The sensors are activated automatically when the patient falls and the help centre is alerted. Thus, second-generation alarms rely on the event and not on the user to initiate the alarm.

\section{The third generation}

Third-generation systems are currently being conceptualised and developed that will directly enhance quality of life, particularly of elderly or disabled people.

It is envisaged that a 'virtual neighbourhood' will be created to replace the actual neighbourhood in which the individual used to live (Doughty et al, 1996). It will consist of houses in geographically diverse locations connected by telecommunications, thereby facilitating communication and interaction. It will be integrated with various teleservices and provide access to banking, shopping, education, marketing, religious services, health services and so on.

\section{Application in dementia}

Dementia is the strongest predictor of the risk of longterm nursing-home placement in old age, increasing it by $54 \%$ (Andel et al, 2007). Additional predictors are listed in Box 5. A major aim of dementia care services is to maintain independence within the home and prevent the need for $24 \mathrm{~h}$ care. Although
Box 5 Predictors of long-term nursing-home placement in patients with dementia

- Functional and cognitive impairment

- Carer burden

- Behavioural and psychological symptoms of dementia

- Incontinence

- Risk of wandering and falls

(Yaffe et al, 2002)

some factors (e.g. cognitive impairment) are not greatly modifiable, others (e.g. the risk of wandering or falls and carer burden) can be minimised with the provision of telecare.

The application of telecare in dementia can be best explained with a few hypothetical examples of common scenarios.

\section{Case vignette 3}

$\mathrm{MrC}$ is an 82-year-old man with a diagnosis of Alzheimer's dementia. He lives on his own and is able to manage his activities of daily living independently. He has help from home care, who supervise his medications. He has a daughter who lives nearby and supports her father in the community. However, in the past 4 months, wandering out of the house has become a problem and he has locked himself out of his house on three occasions. The team treating $\mathrm{Mr} \mathrm{C}$ has suggested that he is admitted to long-term residential care; however, $\mathrm{Mr} \mathrm{C}$ wants to continue to live in his home.

Such a situation is not uncommon in patients with dementia, up to $40 \%$ of whom experience wandering as a problem. The use of a property exit sensor in this case will increase $\mathrm{MrC}^{\prime}$ 's safety, help him to remain living in his own home and reduce his daughter's stress.

A property exit sensor is based on the same technology as a burglar alarm: sensors detect when a door is open. It can be set to raise an alarm only at particular times, for example when the door has been left open for a certain length of time. Once it raises an alarm, the call centre is alerted and an appropriate response is made.

\section{Case vignette 4}

Mr D is an 80-year-old man with Alzheimer's dementia. He lives on his own and is able to manage most of his activities of daily living independently. However, he frequently leaves the gas cooker on without lighting the gas. Other methods have been tried but he struggles with a microwave oven or electric cooker and prefers to use the gas cooker. He also insists on cooking.

This is a common situation and because of the risks involved often leads to long-term placement in 
a nursing or residential home. Here, the use of a gas detector and automatic shut-off device will enable $\mathrm{Mr} \mathrm{D}$ to continue to cook safely. If it detects a high level of gas in the property, the gas detector will raise an alarm at a response centre and the shut-off valve will cut off the gas supply to the cooker. The response centre will contact the carer (or the person named in the response protocol), who can assess the situation and turn on the gas supply again.

\section{Case vignette 5}

Mr E is a 78-year-old man with a diagnosis of Alzheimer's dementia. He lives on his own and has a daughter who lives nearby. He gets up a few times at night to go to the bathroom. On a couple of occasions he forgot to switch on the lights and tripped and fell in the dark. He has also occasionally spent the night in a chair as he forgot to go back to bed.

In this fairly common scenario, a bed occupancy sensor would be particularly useful. It detects when an individual patient leaves their bed and when they come back. So, if Mr E is not back within a particular preset time, an alarm will be raised at the response centre. The sensor can also be set to switch on the lights when MrE leaves his bed, and to switch them off when he returns to it.

The above examples highlight the use of a telecare system in dementia care. However, like any other interventions, there are some barriers to the use of telecare (Box 6).

\section{Ethical issues}

The use of telecare for patients with dementia requires various ethical considerations. Four prima facie moral principles (Box 7) provide a framework for ensuring that these issues have been considered in detail.

\section{Respect for autonomy}

In accordance with the principle of respect for autonomy, professionals have an obligation to respect privacy, ensure confidentiality and obtain informed consent. Every effort must be taken to obtain the patient's informed consent to the use of telecare. However, if the individual lacks capacity to consent the views of people involved in their care, including family, friends and other professionals, should be taken into account and a decision taken in the patient's best interests.

Some telecare systems can intrude into patients' privacy and so before the system is installed it should be decided which aspects need to be monitored and steps should be taken to ensure that privacy is not otherwise invaded. The data generated by
Box 6 Barriers in using telecare with dementia

- Problems in obtaining informed consent regarding the use of telecare

- The attitude prevalent among professional and informal carers that human care is the only appropriate form of support

- Difficulty in identifying appropriate technology for each individual owing to a lack of understanding of dementia

- Assessment that fails to identify specific risk factors in the accommodation of people with dementia, owing to a lack of understanding of the impact of dementia on the individual

- Relative and carer anxiety about the use of technology

the system should be subject to strict confidentiality guidelines. Pilot projects have suggested that it is not the form of technology that determines the ethics of its use, but how it is used in an individual case (Gillies, 2001).

\section{Beneficence and non-maleficence}

The principles of beneficence and non-maleficence require that active steps be taken to ensure that the use of telecare does not cause a patient to lose skills by being overreliant on the system and that it does not end up as an inappropriate substitute for human care. Telecare should be provided as part of a community care package, rather than as a standalone service.

\section{Justice}

Telecare will not be the right solution for every patient and unless a particular telecare system offers a clear benefit for an individual, it should not be used. A comprehensive assessment for the suitability of telecare should be made on an individual basis and, once fitted, the need for the system should be monitored on a regular basis.

Questions that will help address ethical issues in the use of telecare are listed in Box 8.

\section{Evidence for the use of telecare}

As telecare is a recent development, there are limited studies assessing its effectiveness, and most of these are evaluations of specialist projects in controlled environments. There are no large-scale randomised 


\section{Box 7 Four prima facie $^{1}$ moral principles in medical care (after Beauchamp \& Childress, 1989)}

\section{Respect for autonomy}

Autonomy refers to the capability and right of patients to control the course of their own medical treatment and participate in the treatment decision-making process. The principle of respect for autonomy implies that the patient should be free from coercion in taking a decision to act. It also means that professionals have an obligation to respect patients' privacy, ensure patient confidentiality and not deceive the patient, i.e. to tell the truth unless the patient clearly wishes to be deceived. This principle forms the basis of informed consent in clinical practice.

\section{Beneficence}

The principle of beneficence refers to the duty of healthcare providers to be of benefit to the patient, as well as to take positive steps to prevent and to remove harm from the patient.

\section{Non-maleficence}

Non-maleficence refers to the Hippocratic duty of primum non nocere, commonly translated as 'first do no harm'. In clinical practice, there is often a conflict between the principles of beneficence and non-maleficence. This dichotomy is the origin of risk-benefit analysis in clinical practice.

\section{Justice}

Justice means giving each person what they deserve, or, in more traditional terms, giving each person their due. It is often defined as a form of fairness. Aristotle defined the principle of justice as 'equals should be treated equally and unequals should be treated unequally in proportion to the morally relevant inequalities'. People have argued ever since about the morally relevant criteria for regarding and treating people as equals or as unequals.

1. A prima facie principle is always binding unless it conflicts with another prima facie principle, in which case a choice has to be made. Hence, the 'actual duty' of a professional is determined by balancing all the competing prima facie principles in a particular situation.

Box 8 Questions to ask to determine the ethical use of telecare

- Does the patient understand what the telecare system is supposed to do?

- Have they been given a full explanation of the options?

- Have they agreed to try telecare?

- Have their closest family and friends been involved in these discussions?

- Have you considered how you will balance any tension or conflict between the rights and risks of all parties involved?

- Have you used a suitable assessment tool to identify need?

- Have you planned how to introduce the telecare system to the person?

- Have you planned how the telecare will be installed?

- Have you a protocol for its use?

- Have you people who can respond in case of difficulties, emergencies, etc.?

- How will you assess the system's immediate usefulness?

- How will you review its continued usefulness?

- Have you a decommissioning plan?

(Telecare Learning and Improving Network, 2005a) controlled trials assessing the impact of telecare on users and carers.

There are a few projects that have been designed to assess the role of telecare in patients with dementia. The ENABLE project (Enabling Technologies for People with Dementia, 2004) was carried out in five countries and concluded that the stage of the patient's disease and their cognitive functioning were key factors affecting the degree of use and usefulness of the product. For carers, their motivation, willingness and ability to assist the care recipient in using the devices were significant contributory factors. The role and attitudes of professional health and care personnel were also important.

Another project (Woolham, 2006), in the English county of Northamptonshire, showed that in the course of 21 months, nearly $£ 1.5$ million was saved by the use of telecare. It also showed a reduction in carer stress, delay in going into long-term care and improved confidence levels in patients.

Although the evidence is not as robust as would be expected in healthcare, a report funded by the Department of Health to look into the evidence base for telecare (Telecare Learning and Improving Network, 2006) argued that evidence-informed practice is recognised as acceptable in social care (as distinct from evidence-based practice in healthcare). It also stated that evidence from studies that use different approaches to evaluation can be valid provided that their strengths and limitations are explained and understood by those who use it. 
The report concluded that telecare is an emerging type of care delivery and that there is sufficient evidence to assist in setting up and implementing new trials and schemes.

\section{Telecare in the UK}

In July 2004, the government announced its plan to invest $£ 80$ million in telecare services over 2 years from April 2006, through the Preventative Technology Grant (Department of Health, 2005). The aim is to help 160000 older people to live at home in safety and security, and reduce the number of avoidable admissions to residential/nursing-care homes and hospitals. This grant is allocated to all local authorities in England with social service responsibilities. The councils are expected to invest in telecare to help support individuals in the community.

Although guidelines on the implementation of telecare have been published (Telecare Learning and Improving Network, 2005b), local authorities can choose how to implement it in their area. Usually, a particular person in social services (most often an occupational therapist) takes referrals from healthcare professionals and from social services for an assessment and provision of telecare. Although anyone from health services can make a referral, in the context of a community mental health team it is likely that a referral will be made by an occupational therapist after a thorough assessment has been made and deficits identified.

\section{Conclusions}

Telecare provides a new technological way of monitoring and decreasing risks of vulnerable patients in the community. Products are already available that can do this and there is likely to be an expansion in this field. Telecare helps people with dementia to remain independent and is likely to be more cost-effective than long-term residential care.

\section{Declaration of interest}

None.

\section{References}

Andel, R., Hyer, K. \& Slack, A. (2007) Risk factors for nursing home placement in older adults with and without dementia. Journal of Aging and Health, 19, 213-228.

Beauchamp, T. L. \& Childress, J. F. (1989) Principles of Biomedical Ethics (3rd edn). Oxford University Press.

Department of Health (2005) Building Telecare in England. Department of Health (http://www.dh.gov.uk/en/Publications andstatistics/Publications/PublicationsPolicyAndGuidance/ DH_4115303).
Doughty, K., Cameron, K. \& Garner, P. (1996) Three generations of telecare of the elderly. Journal of Telemedicine and Telecare, 2, 71-80.

Enabling Technologies for People with Dementia (2004) Crossnational Analysis Report. ENABLE (http://www.enable project.org).

Fisk, M. J. (2003) Social Alarms to Telecare: Older People's Services in Transition. The Policy Press.

Gillies, B. (2001) Smart Support at Home. An Evaluation of Smart Technology in Dispersed Housing. Livingstone.

Integrating Community Equipment Services (2003) Telecare. Department of Health.

Integrating Community Equipment Services (2004) Telecare. Getting Started. Department of Health (http: / / www.integrated carenetwork.gov.uk/_library/Resources/Housing/Housing_ advice/Telecare_-_Getting_Started.pdf).

Royal Commission on Long Term Care (1999) With Respect to Old Age: Long Term Care - Rights and Responsibilities. TSO (The Stationery Office).

Telecare Learning and Improving Network (2005a) Factsheet: Telecare and Ethics. Department of Health (http://icn.csip. org.uk/telecare/index.cfm?pid=541\&catalogueContentID= 1139).

Telecare Learning and Improving Network (2005b) Telecare Implementation Guide. Department of Health (http://www. integratedcarenetwork.gov.uk/telecare/index.cfm?pid =293).

Telecare Learning and Improving Network (2006) Telecare Evidence. Department of Health (http:/ / icn.csip.org.uk/telecare/index. cfm?pid=541\&catalogueContentID=1110).

Woolham, J. (2006) Safe at Home. The Effectiveness of Assistive Technology in Supporting the Independence of People with Dementia. Hawker Publication.

Yaffe, K., Fox, P., Newcomer, R., et al (2002) Patient and caregiver characteristics and nursing home placement in patients with dementia. JAMA, 287, 2090-2097.

\section{MCQs}

1 Telecare:

a is a subset of assistive technology

$\mathrm{b}$ is the same as telemedicine

c does not involve ethical considerations

$\mathrm{d}$ is not being introduced in the UK

e has no role in dementia care.

2 The second generation of telecare:

a is less proactive than the first generation

$b$ is based on an event occurring rather than activation by the user

c includes the social alarm

$\mathrm{d}$ is of no use for patients with dementia

$\mathrm{e}$ is widely used in the UK.

3 With regard to research in telecare:

a there is evidence that it is cost-effective in the long term

b numerous randomised controlled trials have studied its effectiveness

c the ENABLE project was carried out in three countries

d there is no evidence that it reduces carer stress

e it is established that it is not an useful intervention.

4 With regard to the ethical use of telecare in dementia:

a there is no role for informed consent

b there should be strict confidentiality guidelines 
c telecare is a good substitute for human contact and care

d it is a solution for everybody and hence a comprehensive assessment is not required

e views of friends and family are irrelevant if the patient cannot give an informed consent.

5 In the UK:

a the government announced a plan to invest $£ 80$ million over 2 years

b only an occupational therapist in a community mental health team can make a referral for assessment for telecare

c local authorities have no role in the provision of telecare d there are no telecare implementation guidelines from the Department of Health

e social workers cannot access telecare directly.

\section{MCQ answers}

$\begin{array}{lllllll}1 & & \text { 2 } & & 3 & & \\ \text { a } & \text { T } & \text { a F } & \text { a T } & \text { a F } & \text { a T } \\ \text { b F } & \text { b T } & \text { b F } & \text { b T } & \text { b F } \\ \text { c F } & \text { c F } & \text { c F } & \text { c F } & \text { c F } \\ \text { d F } & \text { d F } & \text { d F } & \text { d F } & \text { d F } \\ \text { e F } & \text { e F } & \text { e F } & \text { e F } & \text { e F }\end{array}$

